\title{
357 TORIPALIMAB PLUS CHEMORADIOTHERAPY IN PATIENTS WITH RECURRENT OR METASTATIC CERVICAL CANCER: A MULTICENTER, OPEN-LABEL, SINGLE-ARM, PHASE II TRIAL
}

${ }^{1}$ Xiaoting $\mathrm{Xu}^{*}$, ${ }^{2}$ Jian Huan, ${ }^{3} \mathrm{Hui}$ Miao, ${ }^{4} \mathrm{Hao}$ Wang, ${ }^{1}$ Yue Wang, ${ }^{2}$ Hongyu Zhu, ${ }^{4}$ Jun Jiang, ${ }^{5}$ Juying Zhou. 'The First Affiliated Hospital of Soochow, Suzhou, China; ${ }^{2}$ The Affiliated Suzhou ScienceandTechnology, Suzhou, China; ${ }^{3}$ Xuzhou Cancer Hospital, Suzhou, China; ${ }^{4}$ The First Affiliated Hospital of Anhui, Hefei, China; ${ }^{5}$ The First Affiliated Hospital of Soochow University, Su Zhou, China

Background Recurrent or metastatic cervical cancer patients who progressed after standard therapy have limited treatment options and poor prognosis with a 1-year survival rate ranging between $15 \%$ and $20 \%$. This study evaluates the efficacy and safety of toripalimab plus chemoradiotherapy in patients with recurrent or metastatic cervical cancer (Clinical trial ID: ChiCTR2000029068)

Methods In this open-label, single-arm, phase 2 study conducted at four radiotherapy centers in East China, eligible patients were confirmed by pathology and/or imaging for recurrent or metastatic cervical cancer. According to the firstline therapies for cervical cancer recommended by NCCN guidelines, all patients were received paclitaxel plus cisplatin regimen, with or without bevacizumab, combined with radiotherapy. After seven fractions radiotherapy at the recurrent or metastatic regions, $240 \mathrm{mg}$ toripalimab every three weeks for six cycles or more were given in combination.

Results Between Jan 14th, 2020, and May 1st, 2021, 24 patients were enrolled. All patients were staged at the first visit, as seven patients were with FIGO (2018) stage I, 10 with stage II, 2 with stage III, 1 with stage IV, and 2 with unclear stage. Of 24 included patients, 22 (91.67\%) had squamous cervical cancer. The median age was 55 (range, 33-72) years. As of May 31, 2021, median follow-up time was 8.5 months [95\% CI: 2.3-10.1]. 14 (58.3\%) of 24 patients who achieved an objective response, including 10 (41.7\%) complete response (CR) and $4(16.7 \%)$ partial response (PR). The median duration of response was not reached and 7 (29.1\%) patients continued toripalimab treatment after the previous 6cycle immunotherapy. The disease control rate was 75\% (18/ 24). Median progression-free survival (PFS) was 8.61 months (95\% CI: 4.14-not reached). For subgroup analysis, the median PFS was significantly prolonged in the CR/PR group compared with that in the SD/PD group [not reached $(95 \%$ CI: 6.21-not reached) versus 5.5 months (95\% CI: 2.69$6.870), \mathrm{P}=0.023]$. There was no significant difference in the median PFS between patients who previously received radiotherapy (8.61 months) and those who didn't (6.87 months) ( $\mathrm{P}$ $=0.641) .8(33.3 \%)$ patients had grade 3-4 treatment-related adverse events (TRAEs). The most common grade 3-4 TRAEs were myelosuppression (29.2\%), hypertriglyceridemia (8.3\%), hypoalbuminemia (4.2\%), pneumonia $(4.2 \%)$, and hypercholesterolemia $(4.2 \%)$.

Conclusions Toripalimab plus chemoradiotherapy showed promising antitumor activity and tolerable toxicities in patients with recurrent or metastatic cervical cancer.

http://dx.doi.org/10.1136/jitc-2021-SITC2021.357 